\title{
The liver's new bud
}

\section{By Benjamin Boettner, Assistant Editor}

Yokohama City University and Sekisui Medical Co. Ltd. researchers have produced vascularized human liver tissue that displays promising metabolic and regenerative capacity in mice. ${ }^{1}$ The team now aims to scale up the procedure to generate bigger tissue segments and will test the treatment for liver failure in larger animals.

The human liver has the distinct capacity to regenerate itself. However, acute liver failure or chronic liver damage, which can stem from alcohol abuse, $\mathrm{HBV}$ or $\mathrm{HCV}$, can reduce the amount of functional liver tissue to less than $10 \%-15 \%$ of the original functional liver mass.

Below that threshold, intrinsic regeneration does not occur. ${ }^{2}$ This point of no return, paired with the relative scarcity of transplantable livers, has spurred research into generating liver tissue de novo that does not rely on functional tissue remaining in patients.

Up to now, efforts to regenerate liver tissue have focused on two main strategies. One is the infusion of either autologous bone marrowderived progenitor cells or G-CSF (CSF3). The former differentiate into mature hepatocytes, whereas the latter promotes the recruitment of bone marrow or blood-derived progenitor cells to the liver.

Phase I trials of both methods have shown improvements in patients with liver cirrhosis. ${ }^{3}$

The other strategy is implantation of more complex, small liver segments generated on artificial or decellularized support structures.

"In contrast to previous attempts, our approach basically reconstitutes the multiple cellular interactions that are important for liver organogenesis, enabling the cells to self-organize into a three-dimensional tissue presumably emulating a natural spatiotemporal orientation." -Takanori Takebe, Yokohama City University Studies by multiple academic groups have shown that recellularized grafts can be implanted into rodents. ${ }^{3,4}$

For both strategies, the drawbacks are short survival times of transplanted cells and structures because of their inability to establish long-lived vascular connectivity and failure to integrate with the host liver.

Now, Takanori Takebe and Hideki Taniguchi, both professors at Yokohama City University, have taken a fresh approach to the problem and created fully vascularized and metabolically active human liver buds in vitro.

The authors turned to induced pluripotent stem (iPS) cells and differentiated them into fetal hepatocytes. ${ }^{5,6}$ Coculturing iPS cells with human endothelial cells and mesenchymal stem cells (MSCs) gave rise to self-organizing structures that resembled the first liver buds in the developing human and mouse embryo.

Comparative gene expression analysis revealed largely overlapping patterns between the artificially generated buds and the embryo tissues, underscoring their developmental similarity.

After transplantation into mice, the microvasculature of the liver buds connected to the host vascular system, which enabled the structures to mature into adult, long-lived hepatic tissue capable of performing metabolic reactions. Implantation of 12 liver buds into the mouse mesentery, a well-perfused tissue in the abdominal cavity, extended the survival of animals with failing livers.

"In contrast to previous attempts, our approach basically reconstitutes the multiple cellular interactions that are important for liver organogenesis, enabling the cells to self-organize into a threedimensional tissue presumably emulating a natural spatiotemporal orientation," said Takebe. "Upon transplantation, cells undergo efficient differentiation into target cell types, experiencing organogenetic inputs from supporting stromal cells, mechanical load and growth stimuli that spatiotemporally correspond to the original developmental stages in the embryo."

The findings were published in Nature.

The team carried out the research in collaboration with ADME \& Toxicology Research Institute, which is a unit of Sekisui that is focused on custom synthesis and pharmacological testing of compounds.

\section{Long live the liver}

Takebe said the team now plans on running studies in nonhuman primates and hopes to enter the clinic in 7-10 years.

Stephen Badylak, deputy director of the McGowan Institute for Regenerative Medicine at the University of Pittsburgh and a professor in the Department of Surgery at the University of Pittsburgh Medical Center, said the study provides proof of concept that complex liver tissue can be grown from scratch.

Although the liver buds are still too small to perfuse a larger tissue segment with blood and at this point lack bile ducts, he said the findings lay the groundwork for a potential new approach to liver transplants.

Badylak, who also is director of the McGowan Institute's Center for Preclinical Tissue Engineering, is generating liver tissue ex vivo using decellularized scaffolds as a growth platform for differentiated adult hepatocytes.?

"In moving toward the clinic, larger animal models will be essential. It will be important to demonstrate the growth potential, vascular development, bile duct formation and required duration for the iPS cell-derived liver buds to sufficiently enable large liver structures to develop in vivo," said Jeffrey Ross, VP of product development at Miromatrix Inc.

Miromatrix has licensed worldwide, exclusive rights to numerous patent applications and issued patents from the University of Minnesota covering methods of perfusion, decellularizing and recellularizing organs and tissues.

Shay Soker, a professor of regenerative medicine at the Wake Forest 
Institute for Regenerative Medicine, said Takebe's team already has addressed two major challenges: identifying a cell source for liver tissue engineering and having it vascularized in vivo. According to Soker, the main remaining hurdle is expanding the size of the liver organoids by $100-1,000$-fold while still adequately perfusing the growing tissue mass.

Soker, Ross and Stuart Forbes, a professor of transplantation and regenerative medicine at The University of Edinburgh, all think a strategy for achieving balanced growth of the liver bud cell types could be combining the Japanese team's approach with scaffold technology. According to Soker, decellularized matrices generated from adult liver tissue could be seeded with liver bud germinal centers that may expand and coalesce into a larger, stable structure with improved perfusion and longevity.

In addition to vascularity, larger liver buds will require balanced growth of all three cellular components-differentiating liver cells, endothelial cells and MSCs. On top of that, the organoid's overall structure and functionality will have to be maintained for a long time after implantation.

Forbes thinks the MSC component of the liver buds could be unpredictable. "Ensuring that mesenchymal cells behave how you want them to is a challenge. Would they grow disproportionately faster than hepatocytes when cultured over longer periods of time?" he asked.

He also noted that cells in a chronically damaged liver are permanently exposed to inflammatory signals that may promote unwanted overgrowth of stromal components of liver buds. "We do not know how stable iPS cell-derived cells will be in vivo long term, particularly in the setting of ongoing liver injury," said Forbes.

Thus, Forbes thinks acute liver failure might be a better fit for the technology than chronic liver disease. In that setting, he said, "the patient's own liver may regenerate if provided with support during a critical period."

\section{Bud screening}

In addition to providing a starting point for developing a nextgeneration approach to liver transplants, functional liver buds could provide a platform for drug and toxicity screening.

Indeed, Takebe and Taniguchi administered ketoprofen and debrisoquine to mice with transplanted liver buds and observed metabolic reactions specific to human liver cells. The two drugs are metabolized differently by human and mouse hepatocytes and thus allowed the researchers to distinguish the activity of transplanted human hepatocytes from that of the mouse liver's hepatocytes.

According to Salman Khetani, cofounder of Hepregen Corp., a company developing complex liver cell platforms suitable for high throughput metabolic testing, comprehensive mouse work with a much larger panel of drugs being metabolized by different enzyme systems will be required to find out whether human liver bud activity can be sufficiently differentiated from the mouse liver's metabolism in vivo.
Thus, he thinks a more immediate application of liver buds might be their use for in vitro drug and toxicity testing.

Badylak also said, "iPS cell-derived liver buds could be generated from not only one human genetic background but also a number of different individual sources. When applied to drug and toxicity testing, such an approach could greatly help to gauge person-to-person variability."

Finally, the approach suggests that regenerative approaches to other organs could be spurred on by iPS cell-derived organ bud technology. As Miromatrix's Ross put it, "Recreating the organ bud-like structure certainly has potential for other organ systems, as it elegantly capitalizes on the very early developmental stage when the morphogenesis of the organ is first put into motion. This could prove an important step in accessing key cell types for future therapies."

Soker specified that mostly modular organs like the liver, pancreas and kidney could benefit from the approach because of their sectional packaging and connectivity to the circulation.

Takebe told SciBX that the team has started to evaluate the applicability of the method to the pancreas and kidney.

Takebe and Taniguchi have filed for a patent covering the methodology for generating organ buds for different organs. The method is available for licensing from Yokohama City University.

Boettner, B. SciBX 6(29); doi:10.1038/scibx.2013.746

Published online Aug. 1, 2013

\section{REFERENCES}

1. Takebe, T. et al. Nature; published online July 3, 2013; doi:10.1038/nature12271

Contact: Takanori Takebe, Yokohama City University, Yokohama, Japan

e-mail: ttakebe@yokohama-cu.ac.jp

Contact: Hideki Taniguchi, same affiliation as above e-mail: rtanigu@yokohama-cu.ac.jp

2. Starzl, T.E. et al. Am. J. Surg. 129, 587-590 (1975)

3. Booth, C. et al. World J. Gastroenterol. 18, 6926-6934 (2012)

4. Wertheim, J.A. et al. Curr. Opin. Organ Transplant. 17, 235-240 (2012)

5. Si-Tayeb, K. et al. Hepatology 51, 297-305 (2010)

6. Espejel, S. et al. J. Clin. Invest. 120, 3120-3126 (2010)

7. Soto-Gutierrez, A. et al. Tissue Eng. Part C Methods 17, 677-686 (2011)

\section{COMPANIES AND INSTITUTIONS MENTIONED}

Hepregen Corp., Medford, Mass.

McGowan Institute for Regenerative Medicine, Pittsburgh, $\mathrm{Pa}$. Miromatrix Inc., Minneapolis, Minn.

Sekisui Medical Co. Ltd., Tokyo, Japan

The University of Edinburgh, Edinburgh, U.K.

University of Minnesota, Minneapolis, Minn.

University of Pittsburgh, Pittsburgh, Pa.

University of Pittsburgh Medical Center, Pittsburgh, Pa. Wake Forest Institute for Regenerative Medicine, WinstonSalem, N.C.

Yokohama City University, Yokohama, Japan 